\title{
Induction chemotherapy with TPF (Docetaxel, Carboplatin and Fluorouracil) in the treatment of locally advanced squamous cell carcinoma of the head and neck
}

\author{
J.D. SCHULTZ, G. BRAN, C. ANDERS, H. SADICK, A. FABER, K. HÖRMANN and A. SAUTER
}

Department of Otorhinolaryngology, Head and Neck Surgery, University Hospital Mannheim, D-68167 Mannheim, Germany

Received April 9, 2010; Accepted May 27, 2010

DOI: $10.3892 /$ or_00000974

\begin{abstract}
Squamous cell carcinoma of the head and neck (SCCHN) presents at a locally advanced (LA) stage in many patients. Chemotherapy, which is one fundamental therapy mode for local disease control of inoperable disease or if organ preservation is desired, has become an important factor of first line treatment regimens either during or prior to radiotherapy (RT). Patients with locoregionally advanced inoperable, recurrent or metastatic disease still have a poor prognosis, which enforces the need for new treatment approaches and new drug therapies, adjusted to the different settings of the disease. One innovative progress for this collective of patients with locally advanced tumor was the implementation of Docetaxel in chemotherapeutic regimes in optimal combination with concurrent chemoradiotherapy or in neoadjuvant setting of induction phase treatment. Docetaxel combined with the conventional chemotherapy regimen, containing Cisplatin and 5-Fluorouracil (TPF), is now acknowledged as being the gold standard of induction treatment. Various studies suggest survival advantage due to the induction chemotherapy (ICT) followed by chemoradiotherapy, which is known as sequential therapy, over chemoradiotherapy alone. In contrast to prevailing studies we administered Docetaxel, Carboplatin and 5-FU within the frame-work of induction chemotherapy instead of conventional use of Cisplatin for five patients with locoregionally advanced HNSCC. The clinical progress was evaluated through cross section imaging (computer tomography/MRI) prior and after ICT and classified following the RECIST criteria. Due to a very small collective of patient and the administration of Carboplatin instead of Cisplatin in this study, it was not possible to document the the efficacy of ICT (TPF) concerning survival advantage in patient with locoregionally advanced head and neck tumors.
\end{abstract}

Correspondence to: Dr J.D. Schultz, Univ.-HNO-Klinik, Universitätsmedizin Mannheim, Theodor Kutzer Ufer 1-3, D-68167 Mannheim, Germany

E-mail: johannes.schultz@umm.de

Key words: induction chemotherapy, Docetaxel, local advanced head and neck carcinoma, Carboplatin, squamous cell carcinoma
Further studies with an extended collective of patients are neccessary.

\section{Introduction}

With a global annual incidence of approximately 644000 cases and 352000 HNSCC associated deaths, squamous cell carcinoma of the head and neck (SCCHN) - which includes carcinomas of the oral cavity, floor of mouth, tongue, tonsils and juxtatonsillar fossae, larynx and pharynx (oropharynx, epipharynx and hypopharynx) - is the fifth most common cancer worldwide $(1,2)$. This tumor entity represents at least $5 \%$ of newly diagnosed cancers in adults in the United States and $8 \%$ worldwide. SCCHN, an aggressive epithelial malignancy, has historically been associated with poor prognosis. Although it is highly curable, detected at an early stage, most of the patients present at a locoregionally advanced disease stage.

After standard therapy for locoregionally advanced disease consisting of definitive chemoradiotherapy, surgery and irradiation in an adjuvant setting or chemoradiotherapy, the overall survival within the next 3 years is only $30-50 \%$. The probability of developing locoregional recurrences or distant metastases is 40-60\% (3-7). Indeed, up until the mid1990s, 5-year survival rates had been reported to be as low as $30 \%$ or below for stage IVa/b (M0) disease (8) and $40 \%$ for stage III disease (9).

Various strategies to improve outcomes by coordinating chemotherapy with surgery, and radiotherapy have been tried. Chemoradiotherapy (CRT) is the standard of care for patients with unresectable SCCHN and organ preservation.

After initial high response rates after chemoinduction regimens, there was little evidence of survival benefit due to the development of locoregional recurrences or devastating outcomes for patients in the clinical trials. Previous studies of induction therapy in resectable and unresectable squamous cell carcinoma of head and neck have suggested that induction chemotherapy is most effective in unresectable disease $(10,11)$. A comprehensive meta-analysis demonstrated for the first time that contribution of induction chemotherapy (neoadjuvant) with Carboplatin and 5-Fluorouracil (PF) improved the rate of survival at 5 years by $5 \%$, as compared with standard radiotherapy plus surgery in patients with locally advanced disease $(12,13)$ with improvement of local/ regional control and reduction of distant metastasis $(10,11)$. These prior results and the introduction of the Taxanes, with 
Table I. Clinical findings after ICT and radiochemotherapy according to RECIST criteria.

\begin{tabular}{|c|c|c|c|c|}
\hline Patients & TPF-cycle & $\begin{array}{l}\text { Response rate after } \\
\text { induction-therapy } \\
\text { (RECIST) }\end{array}$ & $\begin{array}{l}\text { Response rate after } \\
\text { radiochemotherapy } \\
\text { (RECIST) }\end{array}$ & Process \\
\hline 1 & 3 & SD & PD & Exitus letalis \\
\hline 2 & 2 & PD & PD & Palliative chemotherapy \\
\hline 3 & 2 & PR & PD & Palliative chemotherapy \\
\hline 4 & 3 & SD & SD & Follow-up \\
\hline 5 & 3 & SD & PR & Follow-up \\
\hline
\end{tabular}

$\mathrm{SD}$, stable disease; $\mathrm{PD}$, progressive disease; $\mathrm{PR}$, partial remission.

single agent acitivity in recurrent and incurable HNSCC, renewed the interest in induction chemotherapy as a therapeutic option. High response rates in primary sites and longterm survival in curative phase II studies was reported to be $40-80 \%$ in different groups of patients (14-17). Studies of Posner and Vermorken et al showed in the phase III studies TAX 323/324 the improvement of loco-regional control, survival, elimination of distant metastasis, reduced toxicity by implementation of Docetaxel within the ICT regimen compared to PF (18-21).

Posner et al showed a consistent trend towards improved survival regardless of the primary site of the disease, nodal state, primary tumor stage and surgical curability. Recently it has been shown that ICT using the combination of the taxane Docetaxel with Cisplatin, 5-Fluorouracil (5-FU) provides a significant survival benefit over Cisplatin, 5Fluorouracil, when used before either definitive RT (TAX323 trial) or Carboplatin-based concomitant chemoradiotherapy (CCRT), especially in reduction of locoregional failure and organ preservation in larynx and hypopharyngeal tumors. Furthermore, the Tax323 study showed a reduction in toxicity and in mortality of $27 \%$ (18) and a prolongation of the 3 -year survival rate of 37 vs. $26 \%$ for TPF and PF-ICT $(18,20,21)$.

Further investigations in a second large phase III trial TAX 32, including patients with resectable and unresectable locally advanced tumors, demonstrated an absolute improvement in the 3-year survival of $62 \%$ with TPF and $48 \%$ with PF as wells as a reduction in risk of death for ICT with TPF (22). Toxicity was markedly different within the two different ICT regimens. Less mucositis, vomiting and nausea and less hearing loss, fewer toxic deaths were reported within the TPF arm. The TPF ICT was associated with more neutropenia and neutropenic fever (18,20-22).

With this report we want to demonstrate the results of induction chemotherapy with Docetaxel, Carboplatin and 5-Fluorouracil compared to the literature described regimen with Cisplatin in advanced squamous cell carcinoma of head and neck in a primary unresectable condition. This is the first report of Carboplatin in this type of ICT regimen.

\section{Patients and methods}

We report on a collective of five male patients with local advanced squamous cell carcinoma who were treated with three cycle of TPF induction chemotherapy regimens in the period from 12/07 till 10/08. The patients had a median age of 56 , one patient was $>70$ years. The patients had a clinical proven stage of III (1), IV (4), IVa (1), IVb (2), IVc (1) squamous-cell carcinoma, the majority suffered from a carcinoma of the oropharynx (3/5), one in the hypopharynx and one of the oral cavity. Three of the five tumors were unresectable because of tumor fixation, fixed lymph nodes and two because of low surgical curability on the basis of advanced tumor stage (III or IV).

A complete medical history was obtained and tumor assessment was performed at baseline. Tumor responses were assessed by clinical evaluation and imaging studies and were characterized according to modified RECIST criteria (Table I). Toxic effects were assessed daily during induction chemotherapy. According to the RECIST criteria further procedure as primary radiochemotherapy in case of progression of disease or surgical tumor resection, neck dissection after regression of the disease depends on the clinical or imaging evaluation after the induction regimen following ICT. A premedication of $8 \mathrm{mg}$ dexamethasone intravenously was established the day before infusion, on the infusion day and after treatment with Docetaxel (Fig. 1).

\section{Results}

Three of five patients were treated with three cycle of TPF in a period of three weeks. Docetaxel was administered at a dose of $75 \mathrm{mg} / \mathrm{m}^{2}$ (dose per $\mathrm{m}^{2}$ of body-surface area) prior to a $1 \mathrm{~h}$ intravenous infusion of $70 \mathrm{mg} / \mathrm{m}^{2}$ intravenous Carboplatin. Finally the patients were administered $600 \mathrm{mg} / \mathrm{m}^{2}$ Fluorouracil as a continuous 24-h infusion for 4 days.

Overall five male patient were treated with TPF according to the above protocol. Three out of five ICT patients were treated with the complete amount of three cycles of TPF and were classified after clinical and imaging reevaluation as 'stable disease' (SD). For two of the patients a surgical curability afterwards was not possible, which left the combined radiochemotherapy with Carboplatin and 5-Fluorouracil (5-FU) as remaining option. The third patient showed a progredient lymphatic node metastasis of the neck so that a surgical neck dissection had to be performed, followed by a combined RCT. Nevertheless, the progressive tumor disease remained unchanged and the patient died four months after the detection. 


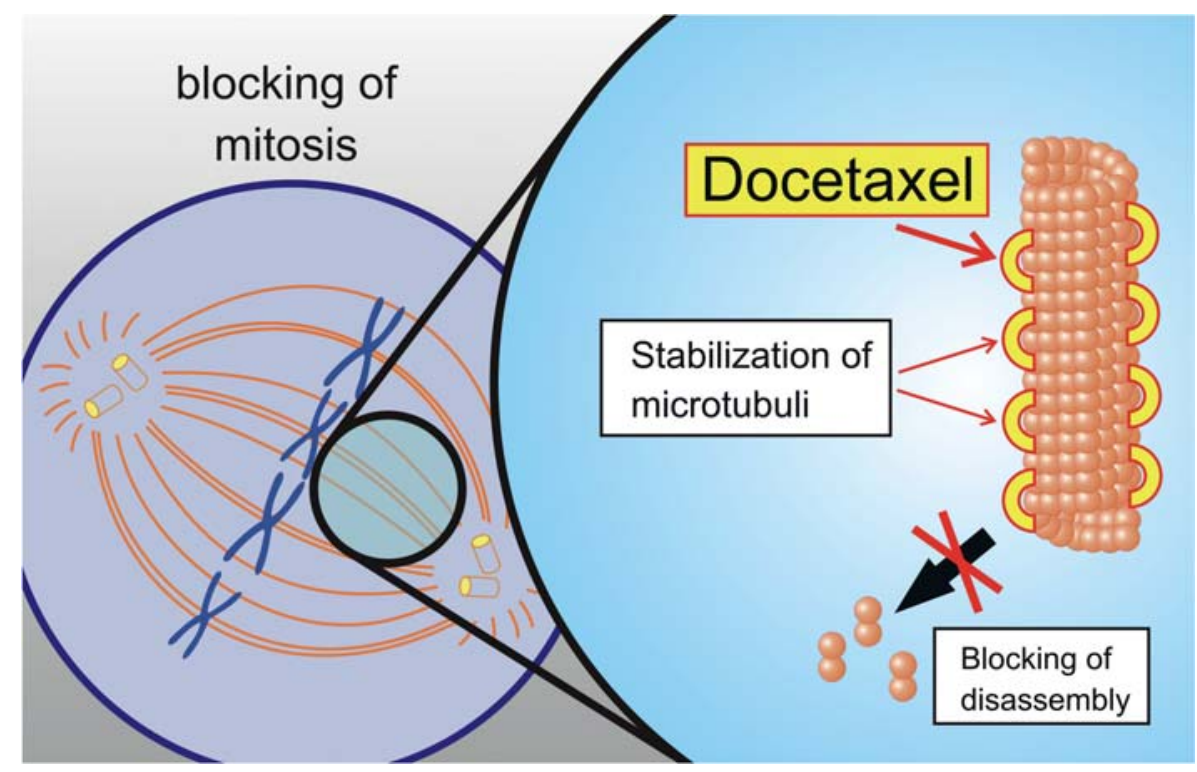

Figure 1. Pathway of Docetaxel interaction in mitosis. Blockage of the depolymerisation of the microtubuli, inducing cell cycle arrest.

Table II. Toxic side effects according to the amount of applied ICT cylces.

\begin{tabular}{lcc}
\hline & Patients & $\begin{array}{c}\text { Event/No. of } \\
\text { cycles (n=13) }\end{array}$ \\
\hline Thrombocytopenia & 1 & $1 / 13$ \\
Anemia Grade III & 1 & $1 / 13$ \\
Neutropenia & & \\
$\quad$ Grade II & 2 & $2 / 13$ \\
$\quad$ Grade III & 2 & $4 / 13$ \\
Febril & 1 & $2 / 13$ \\
Opportunistic infections & 1 & $1 / 13$ \\
\hline
\end{tabular}

For the remaining patients the ICT had to be discontinued after two cycles due to toxic side effects as thrombocytopenia, granulocytopenia, anemia and mucosal local toxicity. One of the patients had a distinct progress of the local advanced tumor under ICT according to the RECIST criteria so that he was treated with combined radiochemotherapy instead of completing the third cycle of TPF. Currently this patient is considered in a state of stable disease and in continuous outpatient control, showing no further progress after this CRT. The second patient who did not complete the three cycles of ICT, suffered from a myocardial infarction (NSTEMI) after second cycle and had to be reanimated subsequently. Further application of the third TPF cycle was not indicated due to delayed convalescence. The discontinuation of treatment was immediately followed by CRT, similarly to the previous patient. Five months after ICT and completed CRT progress of the tumor growth was detected and a palliative chemotherapy initiated. A prediction of efficacy of ICT according to overall survival rate is not yet possible due to the limited observation period and small number of patients.
Toxic side effects. All of the patients that completed the ICT revealed leucopenia. Moreover, two of the patients suffered from multiple leucopenia during the cycles. During/after two cycles there was one case of level II (WHO) neutropenia and four cases of level III (WHO) neutropenia. Thus, 13 cycles were administered for the total collective (Table II).

One patient, who showed an opportunistic infection with a salmonella subspecies, sustained a febrile neutropenia subsequently to a cycle. Overall there was a domination of hematologic complications. In several cases of the neutropenias mentioned before, single or multiple applications of granulocyte colony-stimulating factor (G-CSF) were necessary. Due to pancytopenia there had to be an application of whole blood preservation once. Two patients were given recombinant G-CSF for Docetaxel induced neutrocytopenia.

\section{Discussion}

Our collective showed a lower-order success rate compared to bibliographical reference. One study group reported for instance that $89 \%$ of all biopsies of the primary tumor were negative after three cycles of TPF (22). However, in our study patients received a lower concentration of 5-FU and we administered Carboplatin instead of Cisplatin (18,20-22), which might be a possible reason for the lower response rate of our collective.

Even though prior studies have already shown the efficacy of the combination of Docetaxel, Carboplatin and 5FU in primarily unresectable cancer of the cervix (23) in a non-neoadjuvant context, there are no published studies of induction chemotherapy regimens that consist of this medical drug combination in head and neck cancers. Lu et al compared the short-term efficiency of Docetaxel and Carboplatin versus 5-FU and Carboplatin in local advanced nasopharyngeal tumors and demonstrated that there is no significant difference in short-term efficacy and 1-year survival (24). Even in this study grade 3-4 neutropenia was reported in the Docetaxel/Carboplatin arm. 
The toxicity becomes manifest in hematologic adverse effects. Although the numbers of level III and IV neutropenia were abnormally high in comparison to in-house chemotherapy regimen, Posner et al reported level III and IV neutropenia in the TPF-arm in $84 \%$ of all cases (18). Even in our cases we repeatedly needed to initiate G-CSF and/or the concentrate of erythrocytes. Nevertheless the elevated costs of application of G-CSF have to be discussed.

The application of Docetaxel in advanced head and neck tumors opens up new treatment options in oncological therapy. Initially inoperable tumors, especially oropharyngeal and hypopharyngeal, could be reduced to an operable stage.

As it is well-known, these tumors are characterized by an iniquitous long-term prediction in stage T3 and T4. A reduction of tumor compound through an ICT and follow-up resection could render a considerable advancement of the survival rate. Certainly patients may not have any disadvantage in survival rate due to delayed initiation of radiochemotherapy in persistent inoperability, which will have to be observed. The elevated toxicity of Docetaxel is seen more critically concerning laryngeal cancer. In principle, laryngectomia is a curative but also rather invasive approach of oncological therapy. For this reason, further observations, modifications of chemotherapeutical medication and larger collectives are needed to make path-breaking predications. The application of Docetaxel was operable through an experienced ENToperating department without a considerably higher effort. In our small collective there were no anaphylactic reactions due to application of corticosteroids.

Non-surgical options, using a combination of chemotherapy and radiotherapy, are often used in the treatment of advanced oropharyngeal, hypopharyngeal and laryngeal cancers. Surgery, however, remains an important first-line treatment in certain types of HNSCC (such as oral cavity cancer) and a salvage treatment after failure of radiotherapy with or without chemotherapy. However, successful treatment with curative intent of both LA and M/R SCCHN remains a formidable clinical challenge, and new treatment options and approaches are urgently needed. This is especially true for patients who are either not eligible for surgery, have low surgical curability rates or harbor tumors for which nonsurgical therapy represents a more acceptable, and arguably better, treatment (e.g., RT or chemoradiotherapy for infiltrative LA tonsillar carcinomas). For all the above SCCHN patients, the combination of RT and cytotoxic chemotherapy, and more recently biologically targeted agents, has been the focus of intense clinical investigation, resulting in the launch of several important clinical trials. Further studies with an extended collective of patients are neccessary.

\section{References}

1. Parkin DM, Bray F, Ferlay J, et al: Global cancer statistics. J Clin Cancer 55: 74-108, 2002.

2. IARC (International Agency for Research on Cancer): Globocan. www dep.iarc.fr. 2002.

3. Adelstein D, Li Y, Adams G, et al: An intergroup phase III comparison of standard radiation therapy and two schedules of concurrent chemoradiotherapy in patients with unresectable squamous cell head and neck cancer. J Clin Oncol 21: 92-98, 2003.

4. Denis F, Garaud P, Bardet E, et al: Final results of the 94-01 French Head and Neck Oncology and Radiotherapy Group randomized trial comparing radiotherapy alone with concomitant radiochemotherapy in advanced-stage oropharynx carcinoma. J Clin Oncol 22: 69-76, 2004.

5. Forastiere A, Maor M, Weber R, et al: Long term results of Intergroup RTOG 91-11: a phase III trial to preserve the larynxinduction Cisplatin/5-FU and radiation therapy versus concurrent Cisplatin and radiation therapy versus radiation therapy. Proc Am Soc Clin Oncol 24: abs. 5517, 2006.

6. Bonner JA, Harari PM, Giralt J, et al: Radiotherapy plus cetuximab for squamous-cell carcinoma of the head and neck. N Engl J Med 354: 567-578, 2006.

7. Lefebvre J, Chevalier D, Luboinski B, et al: Larynx preservation in sinus piriformis cancer: preliminary results of a European organization for research and treatment of cancer phase III trial. J Natl Cancer Inst 88: 890-898, 1996.

8. Vokes E: Current treatments and promising investigations in a multidisciplinary setting. Ann Oncol 16 (Suppl. 6): S25-S30, 2005.

9. Laramore GE, Scott CB, Al-Sarraf M, et al: Adjuvant chemotherapy for resectable squamous cell carcinoma of the head and neck: report on Intergroup Study 0034. Int J Radiat Oncol Biol Phys 23: 705-713, 1992.

10. Domenge C, Hill C, Lefebvre J, et al: Randomized trial of neoadjuvant chemotherapy in oropharyngeal carcinoma. Br J Cancer 83: 1594-1598, 2000.

11. Paccagnella A, Orlando A, Marchiori C, et al: Phase III trial of initial chemotherapy in stage III or IV head and neck cancers: a study by the Gruppo di Studio sui Tumori della Testa e del Collo. J Natl Cancer Inst 86: 265-272, 1994.

12. Monnerat C, Faivre S, Temam S, et al: End points for new agents in induction chemotherapy for locally advanced head and neck cancers. Ann Oncol 13: 995-1006, 2002.

13. Pignon JP, Bourhis J, Domenge C, et al: Chemotherapy added to locoregional treatment for head and neck squamous-cell carcinoma: three metaanalyses of updated individual data. Lancet 355: 949-955, 2000.

14. Colevas A, Norris C, Tishler R, et al: A phase II trial of TPFL (Docetaxel, Cisplatin, 5-Fluorouracil, leucovorin) as induction for squamous cell carcinoma of the head and neck (SCCHN). J Clin Oncol 17: 3503-3511, 1999.

15. Colevas AD, Norris CM, Tishler RB, et al: Phase I/II trial of outpatient Docetaxel, Cisplatin, 5-fluorouracil, leucovorin (opTPFL) as induction for squamous cell carcinoma of the head and neck (SCCHN). Am J Clin Oncol 25: 153-159, 2002.

16. Posner MR, Glisson B, Frenette G, et al: Multicentermphase I-II trial of Docetaxel, Cisplatin and Fluorouracil induction chemotherapy for patients with locally advanced squamous cell cancer of the head and neck. J Clin Oncol 19: 1096-1104, 2001.

17. Schrijvers D, van Herpen C, Kerger J, et al: Docetaxel, Cisplatin and 5-fluorouracil in patients with locally advanced unresectable head and neck cancer: a phase I-II feasibility study. Ann Oncol 15: 638-645, 2004.

18. Posner MR, Hershock DM, Blajman CR, et al: Cisplatin and Fluorouracil alone or with Docetaxel in head and neck cancer. N Engl J Med 357: 1705-1715, 2007.

19. Vermorken JB, Remenar E, van Herpen C, et al: Cisplatin, Fluorouracil and Docetaxel in unresectable head and neck cancer. N Engl J Med 357: 1695-1704, 2007.

20. Posner MR, Haddad RI, Wirth L, et al: Induction chemotherapy in locally advanced squamous cell cancer of the head and neck: evolution of the sequential treatment approach. Semin Oncol 31: 778-785, 2004

21. Posner MR and Lefebvre JL: Docetaxel induction therapy in locally advanced squamous cell carcinoma of the head and neck (Review). Br J Cancer 88: 11-17, 2003.

22. Posner MR, Norris CM, Wirth LJ, et al for the TAX 324 Study Group: Sequential therapy for the locally advanced larynx and hypopharynx cancer subgroup in TAX 324: survival, surgery and organ preservation. Ann Oncol 20: 921-927, 2009.

23. Lin JT, Shih SC, Chang TH, et al: Docetaxel, carboplatin and 5fluorouracil (TCF) chemotherapy in patients with unresectable metastatic carcinoma of cerix. Gynecol Oncol 117: 65-69, 2010.

24. Lu X, Guo X, Hong MH, et al: Comparison of the short-term efficacy of two inductive chemotherapy regimens for locally advanced nasopharyngeal caricinoma: docetaxal plus carboplatin versus 5-fluorouracil plus carboplatin. Chin J Cancer 29: 140-144, 2010 . 